\title{
Characterization of genome-wide variations induced by gamma-ray radiation in barley using RNA-Seq
}

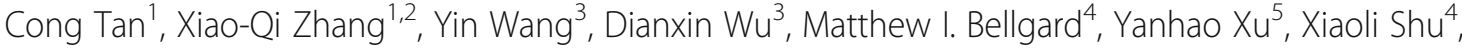 \\ Gaofeng Zhou ${ }^{1,6}$ and Chengdao Li $^{1,3,6^{*}}$
}

\begin{abstract}
Background: Artificial mutagenesis not only provides a new approach to increase the diversity of desirable traits for breeding new varieties but are also beneficial for characterizing the genetic basis of functional genes. In recent decades, many mutation genes have been identified which are responsible for phenotype changes in mutants in various species including Arabidopsis and rice. However, the mutation feature in induced mutants and the underlying mechanisms of various types of artificial mutagenesis remain unclear.
\end{abstract}

Results: In this study, we adopted a transcriptome sequencing strategy to characterize mutations in coding regions in a barley dwarf mutant induced by gamma-ray radiation. We detected 1193 genetic mutations in gene transcription regions introduced by gamma-ray radiation. Interestingly, up to $97 \%$ of the gamma irradiation mutations were concentrated in certain regions in chromosome $5 \mathrm{H}$ and chromosome $7 \mathrm{H}$. Of the 26,745 expressed genes, 140 were affected by gamma-ray radiation; their biological functions included cellular and metabolic processes.

Conclusion: Our results indicate that mutations induced by gamma-ray radiation are not evenly distributed across the whole genome but located in several concentrated regions. Our study provides an overview of the feature of genetic mutations and the genes affected by gamma-ray radiation, which should contribute to a deeper understanding of the mechanisms of radiation mutation and their application in gene function analysis.

Keywords: Artificial mutagenesis, Dwarf mutant, Gamma-ray radiation, Mutation pattern, RNA-Seq, Single nucleic polymorphism

\section{Background}

In the past century, thousands of new crop varieties have been bred from induced mutants and cultivated worldwide [1]. These varieties have played an important role in offering desirable agronomic traits including higher yield, improved quality and abiotic stress tolerance. New varieties derived from gamma-ray radiation mutagenesis have accounted for up to $64 \%$ of all radiation-induced varieties released from 1930 to 2004 [2]. For instance, 'Calrose 76 ', generated by gamma-ray radiation, was the

\footnotetext{
* Correspondence: C.Li@murdoch.edu.au

${ }^{1}$ Western Barley Genetics Alliance, College of Science, Health, Engineering and Education, Murdoch University, Murdoch, WA 6150, Australia

${ }^{3}$ IAEA Collaborating Center, State Key Laboratory of Rice Biology, Zhejiang

University, Hangzhou 310029, China

Full list of author information is available at the end of the article
}

dominant rice variety grown in California (the United of States) until the late 1970 s. 'Calrose 76 ' is about $0.25 \mathrm{~m}$ shorter and has higher potential yield than its parent line 'Calrose' [3, 4]. Meanwhile, artificial mutants have been widely used to characterize the genetic basis of functional genes controlling complex agronomic traits with great importance in crops [5-9]. For example, research showed that the phenotype change from 'Calrose' to 'Calrose 76' was due to a single nucleotide substitution induced by gamma-ray radiation in the exon 2 of the rice 'green revolution gene' sd-1, which encodes a gibberellin 20-oxidase [4]. While mutants are widely used in breeding and genetic research, the molecular feature and underlying mechanisms of artificial mutations remain unclear. 
The most widely used mutant-developing techniques include insertion mutagenesis, chemical mutagenesis and physical mutagenesis [10]. The various mutagenesis technologies have diverse mechanisms and mutation patterns. A comprehensive analysis of flanking sequence tags isolated from a transfer DNA (T-DNA) insertion library, including 31,443 independent transformants, identified that T-DNA insertions were a non-random distribution across the whole genome and occurred more frequently in the distal ends and less so in the centromeric regions [11-13]. Compared with insertion mutagenesis, chemical mutagenesis, e.g., ethyl methanesulfonate (EMS), can induce high-density mutations with random distribution [10]. It has been suggested that EMS causes mispairing through the chemical modification of nucleotides and mostly results in the transition of $\mathrm{C} / \mathrm{G}$ to $\mathrm{T} / \mathrm{A}$ via $\mathrm{C}$-to- $\mathrm{T}$ changing [14-16]. It has also been shown that EMS can result in nucleotide deletion or insertion as well as chromosome fracture [17]. Ionizing radiation is prone to causing chromosome alterations such as deletions and inversions, which is a consequence of repairing ionizing-radiation-induced damage $[18,19]$. Recently, a study on six rice mutants induced by gamma-ray radiation using whole-genome resequencing found that the mutations were more or less evenly distributed across each chromosome [20, 21]. So far, the feature of mutations induced by gamma-ray radiation in barley has not been characterized. With the rapid advancement of technologies in DNA sequencing and molecular biology, it is possible to investigate different mutation types at the whole-genome level in single nucleotide resolution [22-24]. Bulk segregation analysis and restriction site associated DNA sequencing are effective for identifying mutant genes [25-27]. Direct genome sequencing and comparative analysis have also been used to identify causal genes in individual mutants [24] or to characterize all mutations in a large mutant library $[22,23]$. Despite the decreasing cost and advancement of genome sequencing technology, characterizing large and complex genome, such as barley (about 4.83 Gb) $[28,29]$, still poses a serious challenge. In contrast, whole-transcriptome analysis with total RNA sequencing (RNA-Seq) reveal all coding genes and also multiple forms of noncoding RNAs, offering a cost-effective alternative to the whole-genome sequencing for investigating genetic variants in coding regions, in which mutations are likely to lead to the change of phenotype.

In this study, we used whole-transcriptome sequencing to identify genetic mutations in transcription regions which were induced by gamma-ray radiation in a mutant derived from the barley cultivar Vlamingh. We anchored these gamma-ray radiation mutations into annotated gene models, evaluated their genetic effects on the genecoding products, and further investigated the pattern of mutation distribution along chromosomes and the base changes in these genetic mutations. Finally, we predicted the potential functions of genes with coding product changes based on protein sequence similarity. Overall, this study aims to provide insights into the mechanism of mutations induced by gamma-ray radiation, so as to accelerate the effective application of radiation mutagens in exploiting important genetic resources and improving crop breeding.

\section{Results \\ SNPs and InDels screening through transcriptome sequencing}

To understand the feature of genetic mutations induced by gamma-ray radiation, RNA-Seq was performed to investigate the mutations in a gamma-ray radiation mutant (Vla-MT) with dwarf phenotype (Fig. 1a). Vla-MT was derived from the barley cultivar Vlamingh (Vla-WT) irradiated with $200 \mathrm{~Gy}$ gamma rays of around 46,000 barley grains and selected from 10,000 individuals of M2 generation. We focused on genetic mutations in coding regions because most mutant phenotypes were reported to be caused by mutations occurred in the coding region. Two biological replicates of both Vla-MT and Vla-WT were sequenced to avoid negative variants and assess the accuracy of transcriptome sequencing and variant detection.

Raw sequencing data of each sample were first filtered and quality-checked before further bioinformatic processing. As a result, an average of $4.67 \mathrm{~Gb}$ high-quality clean data was retained for each sample, which represents 78.41 folds of total length $(59.56 \mathrm{Mb})$ of 39,733 representative transcripts of all predicted high-confidence genes in barley [28]. The detail information about the data output for each sample was given in Table 1 . The clean reads of each sample were aligned to the barley reference genome with the annotated high-confidence genes as guidance. An average of $95 \%$ of the clean reads in each sample was mapped to the barley Morex reference genome [28], of which $\sim 89 \%$ had unique alignment positions in barley chromosomes. Only the uniquely aligned reads were used to detect SNPs and short InDels for each sample. In theory, in two replicates of each sample, the variants relative to the barley reference should be the same if the sequencing errors and negative-called variants could be avoided. The concordance rate for SNP and InDel calling was $99.98 \%(45,637$ of 45,645$)$ for two replicates of Vla-MT and $99.99 \%(46,582$ of 46,585$)$ for two replicates of VlaWT, indicating that the potential sequencing errors and false positive variant calling were adequately controlled (within $0.01-0.02 \%$ ), and the quality of detected variants was reliable for further investigation of genetic mutations induced by gamma-ray radiation in the mutant. 

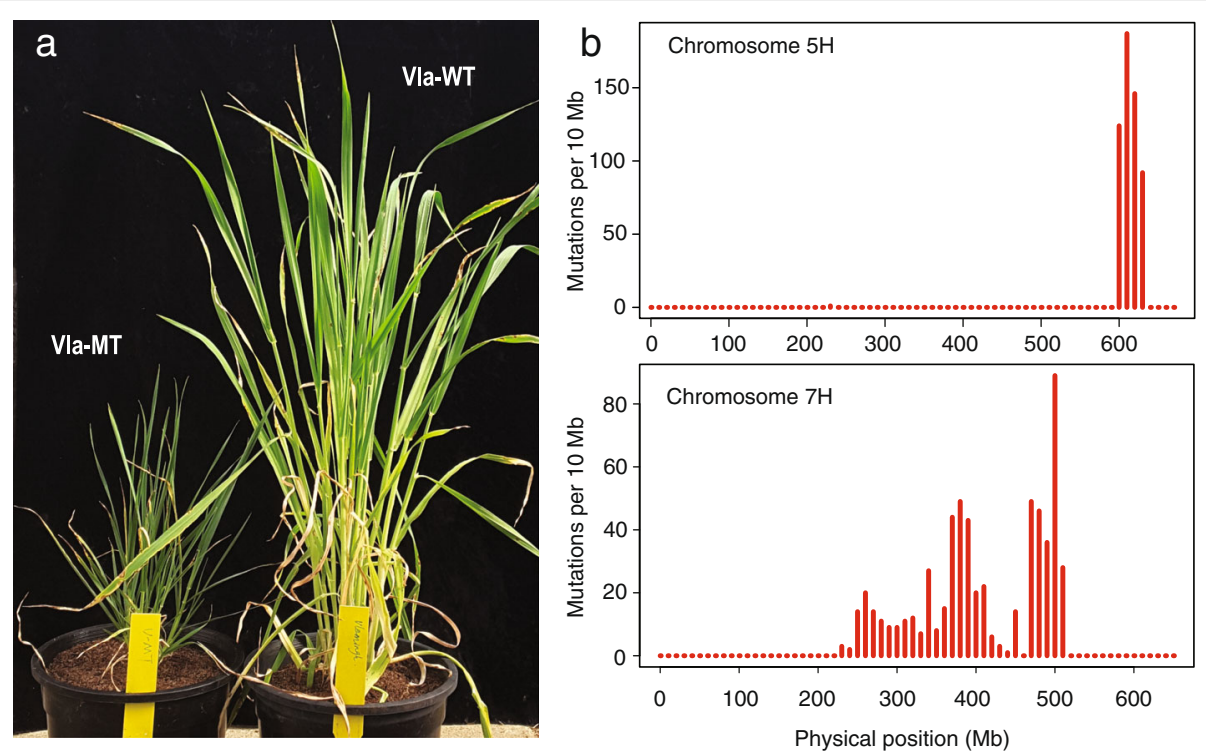

Fig. 1 The phenotype of Vla-WT and Vla-MT and distribution of mutations in chromosome $5 \mathrm{H}$ and chromosome $7 \mathrm{H}$. a, overview of the phenotype of wild-type Vla-WT (right) and mutant type Vla-MT (left). b, distribution of detected mutations in chromosome 5 and 7 . The $x$-axis indicates the physical coordination in chromosome 5 and $\mathrm{Y}$-axis indicates the mutation numbers for each $10 \mathrm{Mb}$ region

\section{Genetic mutations induced by gamma-ray radiation}

Vla-MT was derived from Vla-WT by exposing it to gamma-ray radiation. Hence, the sequence differences between Vla-MT and Vla-WT are assumed to be genetic mutations induced by gamma-ray radiation. We aligned the transcriptomic sequences from both Vla-MT and Vla-WT to the Morex reference genome sequence; $97 \%$ $(42,686$ of 43,879$)$ of the variants were identical between Vla-MT and Vla-WT. In the two repeats of Vla-MT and Vla-WT, 1193 variants were consistently detected and assumed to be genetic mutations induced by gamma-ray radiation (Additional file 1 ).

The 1193 genetic mutations induced by gamma-ray radiation were not evenly distributed in each chromosome (Table 2). Instead, these mutations were mainly located on chromosomes $5 \mathrm{H}$ (550 mutations) and 7H (612 mutations), with only three mutations on chromosome $1 \mathrm{H}$ and one each on $2 \mathrm{H}, 4 \mathrm{H}$, and $6 \mathrm{H}$. There was no mutation identified on chromosome $3 \mathrm{H}$. Further analysis showed that the genetic mutations on chromosome $5 \mathrm{H}$

Table 1 Reads count in the process of filtration and alignment

\begin{tabular}{|c|c|c|c|c|c|c|}
\hline \multirow[t]{2}{*}{$\begin{array}{l}\text { Sample } \\
\text { (replicate) }\end{array}$} & \multicolumn{2}{|l|}{ Raw Reads } & \multicolumn{2}{|l|}{ Clean Reads } & \multicolumn{2}{|c|}{$\begin{array}{l}\text { Uniquely } \\
\text { Mapped Reads }\end{array}$} \\
\hline & (n) & (bp) & (n) & $(\%)$ & (n) & (\%) \\
\hline Vla-WT (1) & $54,869,632$ & 90 & $51,817,980$ & 94 & $46,118,002$ & 89 \\
\hline Vla-WT (2) & 55,10 & 90 & $52,013,200$ & 94 & $46,291,748$ & 39 \\
\hline Vla-MT (1) & $54,344,828$ & 90 & $51,385,788$ & 94 & $45,733,351$ & 89 \\
\hline a-MT (2) & $54,698,866$ & 90 & $51,755,362$ & 94 & $46,062,272$ & 89 \\
\hline Total & $219,020,518$ & 90 & $206,972,330$ & - & $184,205,373$ & - \\
\hline
\end{tabular}

and $7 \mathrm{H}$ were concentrated in several regions (Fig. 1b). On chromosome $5 \mathrm{H}$, the 550 genetic mutations occurred in a $40 \mathrm{Mb}$ genome region (chromosome 5H: 590-630 $\mathrm{Mb})$. On chromosome $7 \mathrm{H}$, the mutations spread across a region of $290 \mathrm{Mb}$ (chromosome $7 \mathrm{H}$ : 230-520 Mb). The results suggest that mutations caused by gamma-ray radiation in Val-MT occurred in certain regions of some chromosomes, other than distributed evenly across the whole genome.

Of the 1193 mutations induced by gamma-ray radiation, 96.65\% were SNPs, with the remaining 26 as short deletions and 14 as short insertions. The exact number of base changes is shown in Table 3. Four dominant changes were $A \rightarrow G, C \rightarrow T, G \rightarrow A, T \rightarrow C$.

\section{Genes affected by mutations}

We investigated where the genetic mutations were located by comparing their physical positions with predicted gene models. In theory, they should all be anchored to transcription regions because only mRNA fragments were selected to conduct sequencing in this study. It is interesting to notice that $14 \%$ of the mutations were mapped to the intergenic regions and a further $6 \%$ to the intron regions (Fig. 2a). It is likely that some potential gene-coding regions were not included in the Morex gene annotations or that alternative splicing events occurred between different germplasm accessions. A further $33 \%$ of the detected genetic mutations were anchored to gene exon regions, with the remaining being in upstream, 5'-UTR, 3'-UTR and downstream regions (Fig. 2a, Additional file 2). We further predicted the 
Table 2 Genetic mutation counts in each chromosome

\begin{tabular}{lll}
\hline Chromosome & Length $(\mathrm{Mb})$ & Variants \\
\hline $1 \mathrm{H}$ & 558.54 & 3 \\
$2 \mathrm{H}$ & 768.08 & 1 \\
$3 \mathrm{H}$ & 699.71 & 0 \\
$4 \mathrm{H}$ & 647.06 & 1 \\
$5 \mathrm{H}$ & 670.03 & 550 \\
$6 \mathrm{H}$ & 583.38 & 1 \\
$7 \mathrm{H}$ & 657.22 & 612 \\
Un & 249.77 & 25 \\
Total & 4833.79 & 1193 \\
\hline
\end{tabular}

genetic effects caused by genetic mutations anchored in gene regions based on coding product changing. Figure 2a (pie chart on the right) shows 209 missense mutations, three nonsense mutations, two frameshift mutations, and one disruptive frameshift mutations. The remains are synonymous mutation, which are assumed to cause fewer effects to coding products.

The 215 genetic mutations including missense, nonsense, frameshift, and disruptive frameshift are assumed to be high-level mutations, which are predicted to be within the exon regions to change coding products. These 215 mutations are harbored in 140 out of 26,748 expressed genes (Additional file 3). According to the protein sequence similarity with other well-researched proteins, these 140 genes are annotated with gene ontology. Their functions are classified into three levels being biological processes, molecular function, and cellular components (Fig. 2b). In the biological process level, cellular and metabolic processes dominate the high-level mutation genes, followed by cellular localization and biological regulation. At the molecular function level, binding and catalytic are the two main types of mutation genes. 40 out of the 140 genes with high-level of mutations searched protein hits in KEGG database. According to the KEGG pathway analysis, the 40 hits present in seven major categories of pathways as Additional file 4 . The predominant pathway category for the genes with high-level mutations is metabolic pathways, which is followed by biosynthesis of secondary metabolites and biosynthesis of antibiotics.

Table 3 Base changes of SNPs revealed as mutations

\begin{tabular}{llllll}
\hline Base changes & $>\mathrm{A}$ & $>\mathrm{C}$ & $>\mathrm{G}$ & $>\mathrm{T}$ & Total \\
\hline A $>$ & 0 & 46 & 192 & 25 & 263 \\
C> & 57 & 0 & 66 & 208 & 274 \\
$\mathrm{G}>$ & 211 & 64 & 0 & 33 & 308 \\
$\mathrm{~T}>$ & 38 & 170 & 43 & 0 & 251 \\
\hline
\end{tabular}

\section{Discussion}

Most reported mutants have exhibited changes in qualitative traits such as the single tiller mutant [30] and never flowering mutant [31] in rice and six-rowed mutant in barley [32]. The focus in those studies has been on understanding the gene controlling the target trait. Vla-MT, with increased tillers and reduced plant height (Fig. 1a), was initially the selected qualitative mutant. In this study, we found up to 1193 genetic mutations detected in gene transcription regions of the barley mutant generated by gamma-ray radiation. Moreover, they were concentrated on two major chromosomes rather than distributed evenly across the whole genome, which is in contrast with the previous report [20]. This may be due to the fact that our study just focused on a specific mutant. At levels, the mutations may be more or less evenly distributed in the whole genome. The other possibility is that different crop may have different responses to gamma-ray radiation as the size of one barley chromosome is close to the whole rice genome. Further study is required to clarify if the difference is due to a cropspecific response to radiation or is due to the limited number of mutant in our study. Our results also revealed that the genetic mutations in the two chromosomes were within several small fragments of genomic regions. The results raised the question whether it is possible for the two regions as heterozygous in the wildtype Vlamingh but that was excluded after investigating the variants result in the wild-type Vlamingh using whole-genome shotgun sequencing data with high coverage. Our results suggest that a mutant selected from gamma-ray radiation may exist wider genetic variations for other genes and traits. In other words, it may be possible to improve quantitative traits using gamma radiation, as up to 140 genes were modified from a single mutant in the present study.

Apart from the 26 short deletions and 14 short insertions, SNP mutations accounted for about $96.65 \%$ of the identified mutations, with $\mathrm{A} \rightarrow \mathrm{G}, \mathrm{C} \rightarrow \mathrm{T}, \mathrm{G} \rightarrow \mathrm{A}$, and $\mathrm{T} \rightarrow \mathrm{C}$ being the four major types. The chemical groups in the side chain are prone to damage by gamma-ray radiation, which would then be repaired by the DNA repair mechanism [33-35]. It is likely that some mutations could be repaired and recovered to their original chemical structure, while others changed in their similar chemical structures [34]. The scenario could be the same for cytosine $(\mathrm{C})$ and thymine $(\mathrm{T})$ because they share the same monocycle and differ in two chemical groups of the side chain.

Of the 1193 genetic mutations, 450 were anchored in gene exon regions, with 215 predicted to be high-level genetic mutations that cause disruptive in-frameshift, frameshift, nonsense, and missense mutations. The coding products of 140 genes were predicted to be changed 


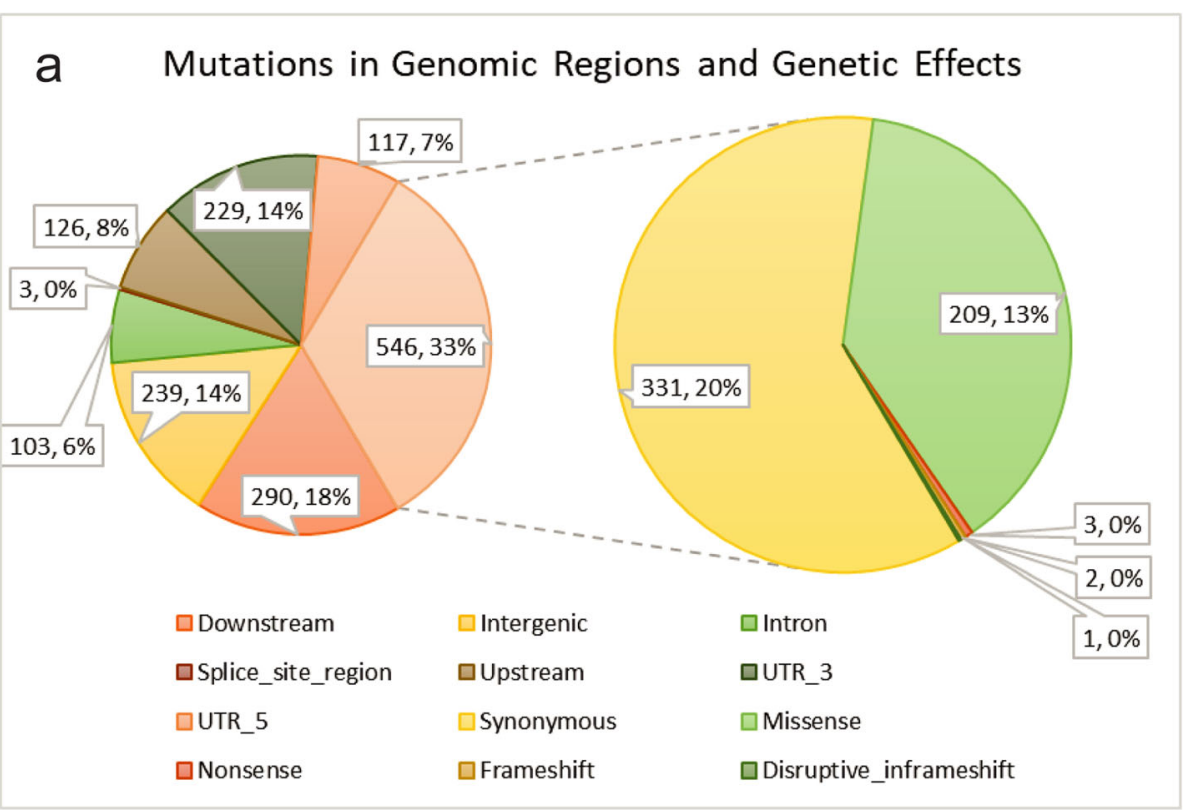

b

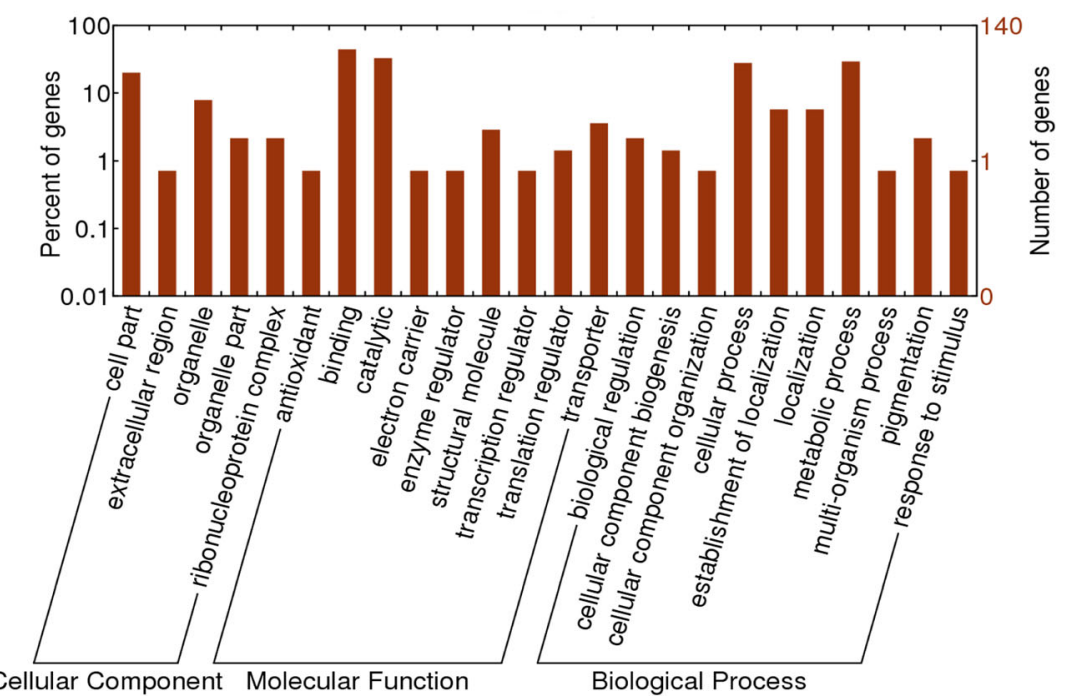

Fig. 2 Genetic effects of mutations and biological function of genes with mutant genes. a, genetic effect prediction of all detected mutations: left pie chart shows the percent of mutations anchored into different types of genomic regions and right pie chart shows the percent of genetic effects of mutations anchored in exon regions. $\mathbf{b}$, biological function classification of affected genes in the cellular component, molecular function, and biological function levels. The $x$-axis indicates the different types of functions and $y$-axis indicates the number and percent of genes in each biological function classification

by the mutation from gamma-ray radiation. This result contrasts with traditional views that only a few genes are affected and responsible for the mutant phenotype [4-6].

While whole-genome resequencing has been used to identify mutations and isolate mutant genes in species with the simple and small genomes in recent years [22, 23], the sequencing cost for large and complex genomes still pose a challenge. Sequencing depth of 30 folds is recommended for reliable variants analysis [36], therefore $150 \mathrm{~Gb}$ data for each sample would be required to investigate mutations in the whole genome for barley $[28,29]$. Most variants related to the loss/change function of gene coding product are in the gene-coding regions. Our study with an average data of $4.6 \mathrm{~Gb}$, equivalent to 78.41 folds of the total length of barley gene transcripts, demonstrated that whole transcriptome analysis provides sufficient reliability and resolution as a cost-effective alternative to whole genome sequencing. Meanwhile, whole transcriptome analysis allows the examination of differential expressed genes between mutant and 
wild plant, which is critical for understanding the genetic and biological basis of mutant genes. Finally, novel transcripts can be detected in transcriptome analysis to improve gene annotation of the reference genome. Variants located in the intron and non-coding regions may be missed in transcriptome sequencing. Although mutations in non-coding regions such as intron region, and intergenic regions, may also play an important role in regulating gene expression level and further leading to phenotype change, the possibility is very low.

Over the past several decades, map-based cloning has played a critical role in identifying genes related to important agronomic traits in crop species and in understanding the genetic basis of plant development in general [37-40]. As molecular marker density has increased dramatically with the application of genotyping-by-sequencing, the current challenge for map-based cloning is population size, which needs to be large enough to produce sufficient recombination events [17, 41]. However, developing a large population to produce sufficient recombination events is laborious and expensive, and is especially challenging for species with large genome size. The present study, through a combination of induced mutation and transcriptome sequencing, provides a complementary strategy to classical bi-parental cross-mapping for the identification of functional genes.

\section{Conclusion}

(1) Our study identified that a large number of genes (140) are affected by gamma-ray radiation in a barley mutant.

(2) The mutations induced by gamma-ray radiation are not evenly distributed in the whole genome, instead, mutations are located in several concentrated regions in certain chromosomes.

(3) Our study provides an overview of the feature of genetic mutations and genes caused by gamma-ray radiation, which should offer a deeper understanding of the mechanisms of radiation mutation and their application in gene function analysis.

\section{Methods}

\section{Plant materials}

Two barley accessions involved in this study are Hordeum vulgare L. cv. Vlamingh (Vla-WT) and one Vlamingh mutant (Vla-MT). Vla-WT is a malt barely variety with high yield, bred by Department of Primary Industry and Regional Development Western Australia. Vla-MT was a dwarf mutant induced by 200 Gry ${ }^{60} \mathrm{Co}$ gamma radiation from around 46,000 M0 dry seeds of Vla-WT and selected from 10,000 M2 individuals. Vla-WT and Vla-MT were sown in growth pots (diameter $45 \mathrm{~cm}$ and $25 \mathrm{~cm}$ in height) in the glasshouse of Murdoch University $\left(31.95^{\circ} \mathrm{S} 115.86^{\circ}\right.$
E, Perth, Australia) in May 2015 (10.5/13.5, D/N). These plants were grown under normal condition and irrigated twice every week (Monday and Thursday). Leave of both Vla-MT and Vla-WT (with two biological replicates) were collected at six weeks old, frozen in liquid nitrogen immediately and stored at $-80^{\circ} \mathrm{C}$.

\section{Transcriptome sequencing}

Total RNA was extracted using TRIzolTM Reagent (Invitrogen, California, the United States) following its user guide. The integrity and purification of RNA samples were qualified using the 2100 Bioanalyzer instrument (Agilent Technologies, California, the United States) and 1.5\% agarose gel. RNA-Seq libraries were constructed following the manufacturer's instruction of TruSeq RNA Library Prep Kit v2 (Illumina, California, United States) and sequenced on the HiSeq 2000 platform in Beijing Genomics Institute-Shenzhen (Shenzhen, China).

\section{Reads mapping and SNP/InDel calling}

Raw reads (90 bp paired-end) produced by the sequencer were filtered to remove reads with low quality using Sickle [42] (version 1.33, parameters as "pe -q 30 -l 50"). The quality of cleaned data was assessed using FASTQC toolkit [43]. Clean reads of each sample were mapped to the latest barley reference genome [28] using the sliced aligner STAR [44] (version 2.5.3, default parameters). PCR duplications were filtered using SAMtools [45] (version 1.4.1, default parameters). Only reads with unique mapping positions in the reference proceeded to call SNPs and InDels for each sample. This step was performed using the pipeline consisting of SAMtools [45] (version 1.4.1, parameters as "samtools mpileup --adjustMQ 50 --max-depth 100 --redo-BAQ --min-MQ 20 --min-BQ 13") and BCFtools [46] (Version 1.5, parameters as "bcftools call $-\mathrm{v}-\mathrm{c}-\mathrm{O} \mathrm{z}$ ").

SNPs and InDels were filtered using the following criteria: (1) variants within the low-complexity regions (LCRs) were removed; (2) variants with calling quality < 50 and mapping quality $<40$ were removed; (3) variants with more than five read numbers supporting the nonreference allele, and a percentage $>20 \%$, were retained; (4) SNPs within $15 \mathrm{bp}$ of the flanking region of an InDel were removed. SNP and InDels filtration was performed using BCFtools [46] (Version 1.5, parameters as "filter -SnpGap 15, IndelGap 15 -e 'MQ<40" ) and VCFtools [47] (Version 0.1.14, parameters as "-minQ 50, --minDP 5, -maxDP 100, -minGQ 20"). The detail processing scripts for these analysis are given as Additional file 5 .

\section{Genetic effects assessment and function annotation for mutations}

The genetic effects of each mutation were predicted based on their position in gene models and the change 
of coding product using SnpEff [48] (Version 4.3, default parameters). The impact effect of each mutation was assessed and classified into four levels: high (including frameshift, nonsense), moderate (including missense), modifier (in intron) and low (synonymous). Those genes with high and moderate impact genetic effects were selected for function and pathway annotation. This step was achieved using AutoFACT [49] (Version 3.4, default parameters) based on the homology of their encoding protein with well-researched genes and proteins stored in databases of NR, Swiss-Prot, COG and GO. KEGG annotation mainly includes two steps: (1) extract $\mathrm{K}$ numbers by searching the KEGG database with protein sequences through the web server (https://www.kegg.jp/blastkoala/); (2) obtain pathway annotation by mapping the extracted $\mathrm{K}$ numbers to KEGG pathway maps using KEGG mapper (https://www.genome.jp/kegg/mapper.html).

\section{Supplementary information}

Supplementary information accompanies this paper at https://doi.org/10. 1186/s12864-019-6182-3.

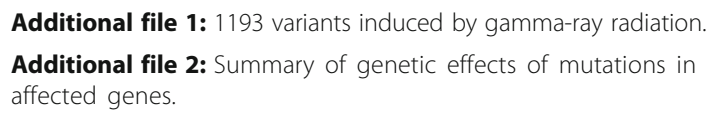

\section{Abbreviations}

COG: Cluster of Orthologous Groups; EMS: ethyl methane-sulfonate; GO: Gene Ontology; InDel: insertion or deletion of bases; KEGG: Kyoto Encyclopaedia of Genes and Genomes; NR: non-redundant protein sequences; RAD-Seq: restriction site associated DNA sequencing; RNASeq: RNA sequencing; SNP: single-nucleotide polymorphism; SwissProt: UniProtKB/Swiss-Prot; Vla-MT: dwarf mutant from cultivar barley Vlamingh in this study; Vla-WT: wild type of cultivar barley Vlamingh

\section{Acknowledgements}

Computing resources were provided by the Pawsey Supercomputing Centre with funding from the Australian Government. Thanks to Dr Tianhua He for his help in proofreading and manuscript revising.

\section{Authors' contributions}

$\mathrm{CL}$ conceived and designed this study and finalized the manuscript. $\mathrm{CT}$ performed the bioinformatic process, interpreted results and penned the draft manuscript. XZ prepared samples and conducted RNA extraction. YW, $\mathrm{DW}, \mathrm{XS}$, and $\mathrm{XY}$ conducted the gamma-ray radiation and selected the mutant. MB organized the computing resource and providing guidance for bioinformatics analysis. GZ attended the discussion about the result interpretation and provide helpful advice. All authors have read and approved the manuscript.

\section{Funding}

This work was supported by the Australian Grain Research and Development Corporation (UMU00050). The funders had no role in study design, data collection and analysis, decision to publish, or preparation of the manuscript.

\section{Availability of data and materials}

Raw data of transcriptome sequencing involved in this study have been deposited in NCBI Sequence Reads Archive with accession number
PRJNA392197 and whole genome shotgun sequencing data of $\mathrm{cv}$. Vlamingh with the accession number SRS1936521.

Ethics approval and consent to participate

Not applicable.

\section{Consent for publication}

Not applicable.

\section{Competing interests}

The co-author Professor Matt Bellgard is a member of editorial board of this journal and he was not involved in the review and editorial process for this paper. The other authors declare that they have no competing interests.

\section{Author details}

'Western Barley Genetics Alliance, College of Science, Health, Engineering and Education, Murdoch University, Murdoch, WA 6150, Australia. 'Western Australian State Agricultural Biotechnology Centre, College of Science, Health, Engineering and Education, Murdoch University, Murdoch, WA 6150, Australia. ${ }^{3}$ IAEA Collaborating Center, State Key Laboratory of Rice Biology, Zhejiang University, Hangzhou 310029, China. ${ }^{4}$ eResearch Office, Queensland University of Technology, Brisbane 4000, Australia. ${ }^{5}$ Hubei Collaborative Innovation Center for Grain Industry, Yangtze University, Jingzhou 434023, Hubei, China. ${ }^{6}$ Western Australia Department of Primary Industry and Regional Development, South Perth, WA 6151, Australia.

Received: 21 April 2019 Accepted: 11 October 2019

Published online: 29 October 2019

\section{References}

1. Ahloowalia BS, Maluszynski M. Induced mutations - a new paradigm in plant breeding. Euphytica. 2001;118(2):167-73.

2. Ahloowalia BS, Maluszynski M, Nichterlein K. Global impact of mutationderived varieties. Euphytica. 2004:135(2):187-204.

3. Rutger JN, Peterson ML, Hu C. Registration of Calrose 76 Rice1 (Reg. No. 45). Crop Sci. 1977:17(6):978.

4. Monna L. Positional cloning of Rice Semidwarfing gene, sd-1: Rice "green revolution gene" encodes a mutant enzyme involved in gibberellin synthesis. DNA Res. 2002;9(1):11-7.

5. Yan H, Saika H, Maekawa M, Takamure I, Tsutsumi N, Kyozuka J, Nakazono M. Rice tillering dwarf mutant dwarf3 has increased leaf longevity during darkness-induced senescence or hydrogen peroxide-induced cell death. Genes Genet Syst. 2007;82(4):361-6.

6. Arite T, Umehara M, Ishikawa S, Hanada A, Maekawa M, Yamaguchi S, Kyozuka J. d14, a strigolactone-insensitive mutant of rice, shows an accelerated outgrowth of tillers. Plant Cell Physiol. 2009;50(8):1416-24.

7. Abe A, Kosugi S, Yoshida K, Natsume S, Takagi H, Kanzaki H, Matsumura H, Yoshida K, Mitsuoka C, Tamiru M, et al. Genome sequencing reveals agronomically important loci in rice using MutMap. Nat Biotechnol. 2012; 30(2):174-8.

8. Arite T, Iwata H, Ohshima K, Maekawa M, Nakajima M, Kojima M, Sakakibara H, Kyozuka J. DWARF10, an RMS1/MAX4/DAD1 ortholog, controls lateral bud outgrowth in rice. Plant J. 2007;51(6):1019-29.

9. Ishikawa S, Maekawa M, Arite T, Onishi K, Takamure I, Kyozuka J. Suppression of tiller bud activity in tillering dwarf mutants of rice. Plant Cell Physiol. 2005;46(1):79-86.

10. Wang N, Long T, Yao W, Xiong L, Zhang Q, Wu C. Mutant resources for the functional analysis of the rice genome. Mol Plant. 2013;6(3):596-604.

11. Wu C, Li X, Yuan W, Chen G, Kilian A, Li J, Xu C, Li X, Zhou DX, Wang S, et al. Development of enhancer trap lines for functional analysis of the rice genome. Plant J. 2003;35(3):418-27.

12. Zhang J, Li C, Wu C, Xiong L, Chen G, Zhang Q, Wang S. RMD: a rice mutant database for functional analysis of the rice genome. Nucleic Acids Res. 2006;34(Database issue):D745-8.

13. Zhang J, Guo D, Chang Y, You C, Li X, Dai X, Weng Q, Zhang J, Chen G, Li $X$. Non-random distribution of T-DNA insertions at various levels of the genome hierarchy as revealed by analyzing 13804 T-DNA flanking sequences from an enhancer-trap mutant library. Plant J. 2007:49(5):947-59.

14. Kim Y, Schumaker KS, Zhu J-K: EMS mutagenesis of Arabidopsis. In: Arabidopsis Protocols. Springer; 2006: 101-103. 
15. Greene EA, Codomo CA, Taylor NE, Henikoff JG, Till BJ, Reynolds SH, Enns LC, Burtner C, Johnson JE, Odden AR, et al. Spectrum of chemically induced mutations from a large-scale reverse-genetic screen in Arabidopsis. Genetics. 2003;164(2):731-40.

16. Koornneeff M, Dellaert $L$, Van der Veen J. EMS-and relation-induced mutation frequencies at individual loci in Arabidopsis thaliana (L.) Heynh. Mutat Res. 1982;93(1):109-23.

17. Sega GA. A review of the genetic effects of ethyl methanesulfonate. Mutat Res. 1984;134(2-3):113-42.

18. Shirley BW, Hanley S, Goodman HM. Effects of ionizing radiation on a plant genome: analysis of two Arabidopsis transparent testa mutations. Plant Cell. 1992;4(3):333-47.

19. Sankaranarayanan K. lonizing radiation and genetic risks. III. Nature of spontaneous and radiation-induced mutations in mammalian in vitro systems and mechanisms of induction of mutations by radiation. Mutat Res. 1991;258(1):75-97.

20. Li S, Zheng YC, Cui HR, Fu HW, Shu QY, Huang JZ. Frequency and type of inheritable mutations induced by gamma rays in rice as revealed by whole genome sequencing. J Zhejiang Univ Sci B. 2016;17(12):905-15.

21. Li S. Liu S-m, Fu H-w, Huang J-z, Shu Q-y: high-resolution melting-based TILLING of $y$ ray-induced mutations in rice. J Zhejiang Univ-Sc B. 2018;19(8):620-9.

22. Li G, Jain R, Chern M, Pham NT, Martin JA, Wei T, Schackwitz WS, Lipzen AM, Duong PQ, Jones KC, et al. The sequences of 1504 mutants in the model Rice variety Kitaake facilitate rapid functional genomic studies. Plant Cell. 2017;29(6):1218-31.

23. Li G, Chern M, Jain R, Martin JA, Schackwitz WS, Jiang L, Vega-Sanchez ME, Lipzen AM, Barry KW, Schmutz J, et al. Genome-wide sequencing of 41 Rice (Oryza sativa L.) mutated lines reveals diverse mutations induced by fastneutron irradiation. Mol Plant. 2016;9(7):1078-81.

24. Nordstrom KJ, Albani MC, James GV, Gutjahr C, Hartwig B, Turck F, Paszkowski U, Coupland G, Schneeberger K. Mutation identification by direct comparison of whole-genome sequencing data from mutant and wild-type individuals using k-mers. Nat Biotechnol. 2013;31(4):325-30.

25. Takagi H, Abe A, Yoshida K, Kosugi S, Natsume S, Mitsuoka C, Uemura A Utsushi H, Tamiru M, Takuno S, et al. QTL-seq: rapid mapping of quantitative trait loci in rice by whole genome resequencing of DNA from two bulked populations. Plant J. 2013;74(1):174-83.

26. Takagi H, Uemura A, Yaegashi H, Tamiru M, Abe A, Mitsuoka C, Utsushi H, Natsume S, Kanzaki H, Matsumura H, et al. MutMap-gap: whole-genome resequencing of mutant F2 progeny bulk combined with de novo assembly of gap regions identifies the rice blast resistance gene Pii. New Phytol. 2013; 200(1):276-83.

27. Nakata M, Miyashita T, Kimura R, Nakata Y, Takagi H, Kuroda M, Yamaguchi T, Umemoto T, Yamakawa H. MutMapPlus identified novel mutant alleles of a rice starch branching enzyme $\mathrm{ll} b$ gene for fine-tuning of cooked rice texture. Plant Biotechnol J. 2018;16(1):111-23.

28. Mascher M, Gundlach H, Himmelbach A, Beier S, Twardziok SO, Wicker T, Radchuk V, Dockter C, Hedley PE, Russell J, et al. A chromosome conformation capture ordered sequence of the barley genome. Nature. 2017:544(7651):427-33.

29. Beier S, Himmelbach A, Colmsee C, Zhang XQ, Barrero RA, Zhang Q, Li L, Bayer M, Bolser D, Taudien S, et al. Construction of a map-based reference genome sequence for barley. Hordeum vulgare L Sci Data. 2017:4:170044.

30. Li X, Qian Q, Fu Z, Wang Y, Xiong G, Zeng D, Wang X, Liu X, Teng S, Hiroshi $F$, et al. Control of tillering in rice. Nature. 2003;422(6932):618-21.

31. Wu C, You C, Li C, Long T, Chen G, Byrne ME, Zhang Q. RID1, encoding a Cys2/His2-type zinc finger transcription factor, acts as a master switch from vegetative to floral development in rice. Proc Natl Acad Sci U S A. 2008; 105(35):12915-20.

32. Komatsuda T, Pourkheirandish M, He C, Azhaguvel P, Kanamori H, Perovic D, Stein N, Graner A, Wicker T, Tagiri A, et al. Six-rowed barley originated from a mutation in a homeodomain-leucine zipper I-class homeobox gene. Proc Natl Acad Sci U S A. 2007;104(4):1424-9.

33. Collins AR. The comet assay for DNA damage and repair: principles, applications, and limitations. Mol Biotechnol. 2004;26(3):249-61.

34. Friedberg EC. DNA damage and repair. Nature. 2003;421(6921):436-40.

35. Rastogi RP, Richa KA, Tyagi MB, Sinha RP. Molecular mechanisms of ultraviolet radiation-induced DNA damage and repair. J Nucleic Acids. 2010;2010:592980.

36. Sims D, Sudbery I, llott NE, Heger A, Ponting CP. Sequencing depth and coverage: key considerations in genomic analyses. Nat Rev Genet. 2014; 15(2):121-32.
37. Xue W, Xing Y, Weng X, Zhao Y, Tang W, Wang L, Zhou H, Yu S, Xu C, Li X, et al. Natural variation in Ghd7 is an important regulator of heading date and yield potential in rice. Nat Genet. 2008;40(6):761-7.

38. Li Y, Fan C, Xing Y, Jiang Y, Luo L, Sun L, Shao D, Xu C, Li X, Xiao J, et al. Natural variation in GS5 plays an important role in regulating grain size and yield in rice. Nat Genet. 2011:43(12):1266-9.

39. Li Y, Fan C, Xing Y, Yun P, Luo L, Yan B, Peng B, Xie W, Wang G, Li X, et al. Chalk5 encodes a vacuolar $\mathrm{H}(+)$-translocating pyrophosphatase influencing grain chalkiness in rice. Nat Genet. 2014;46(4):398-404.

40. Fan C, Xing Y, Mao H, Lu T, Han B, Xu C, Li X, Zhang Q. GS3, a major QTL for grain length and weight and minor QTL for grain width and thickness in rice, encodes a putative transmembrane protein. Theor Appl Genet. 2006; 112(6):1164-71.

41. Wang L, Wang A, Huang X, Zhao Q, Dong G, Qian Q, Sang T, Han B. Mapping 49 quantitative trait loci at high resolution through sequencingbased genotyping of rice recombinant inbred lines. Theor Appl Genet. 2011 ; 122(2):327-40.

42. Sickle: A sliding-window, adaptive, quality-based trimming tool for FastQ files [https://github.com/najoshi/sickle].

43. FastQC: a quality control tool for high throughput sequence data [https:// www.bioinformatics.babraham.ac.uk/projects/fastqc/].

44. Dobin A, Davis CA, Schlesinger F, Drenkow J, Zaleski C, Jha S, Batut P, Chaisson M, Gingeras TR. STAR: ultrafast universal RNA-seq aligner. Bioinformatics. 2013;29(1):15-21.

45. Li H, Handsaker B, Wysoker A, Fennell T, Ruan J, Homer N, Marth G, Abecasis G, Durbin R. Genome project data processing S: the sequence alignment/ map format and SAMtools. Bioinformatics. 2009;25(16):2078-9.

46. Li H. A statistical framework for SNP calling, mutation discovery, association mapping and population genetical parameter estimation from sequencing data. Bioinformatics. 2011;27(21):2987-93.

47. Danecek P, Auton A, Abecasis G, Albers CA, Banks E, DePristo MA, Handsaker RE, Lunter G, Marth GT, Sherry ST, et al. The variant call format and VCFtools. Bioinformatics. 2011;27(15):2156-8.

48. Cingolani P, Platts A, Wang le L, Coon M, Nguyen T, Wang L, Land SJ, Lu X, Ruden DM: A program for annotating and predicting the effects of single nucleotide polymorphisms, SnpEff: SNPs in the genome of Drosophila melanogaster strain w1118; iso-2; iso-3. Fly (Austin) 2012, 6(2):80-92.

49. Koski LB, Gray MW, Lang BF, Burger G. AutoFACT: an auto matic F unctional a nnotation and C lassification T ool. BMC Bioinformatics. 2005;6(1):151.

\section{Publisher's Note}

Springer Nature remains neutral with regard to jurisdictional claims in published maps and institutional affiliations.

Ready to submit your research? Choose BMC and benefit from:

- fast, convenient online submission

- thorough peer review by experienced researchers in your field

- rapid publication on acceptance

- support for research data, including large and complex data types

- gold Open Access which fosters wider collaboration and increased citations

- maximum visibility for your research: over $100 \mathrm{M}$ website views per year

At $\mathrm{BMC}$, research is always in progress.

Learn more biomedcentral.com/submissions 